\title{
Rapid Analysis of Arbitrary-Shaped Conformal Beam-Scanning Arrays
}

\author{
Denys Nikolayev*†, Agnese Mazzinghi ${ }^{\ddagger}$, Anja K. Skrivervik* \\ *Microwave and Antenna Group (MAG), École polytechnique fédérale de Lausanne, \\ CH-1015 Lausanne, Switzerland \\ ${ }^{\dagger}$ Univ Rennes, CNRS, Institut d'Électronique et de Télécommunications de Rennes, UMR-6164, \\ FR-35000 Rennes, France \\ ${ }_{\ddagger}^{\ddagger}$ Department of Information Engineering, University of Florence, IT-50139 Florence, Italy \\ d@deniq.com
}

\begin{abstract}
Conformal antenna arrays are able to fit seamlessly on curved 3D-shaped surfaces, which are found ubiquitously on vehicles, aircraft, a human body, etc. In addition, conformal structures can overcome the scan loss limitations of conventional planar arrays. Yet, only a few computational analysis methods have been proposed with either performance or applicability limitations. In this study, a rapid analysis methodology is developed for the analysis of arbitrary-shaped conformal beamscanning arrays. The method requires only specifying the element position vectors and normals in space. Arbitrary individual antenna patterns could be specified. Finally, an example study is provided examining the beam-steering performance of halfcylinder conformal arrays vs a reference square planar array.
\end{abstract}

Index Terms-conformal array, beam scanning, directivity, scan loss, rapid code.

\section{INTRODUCTION}

Conformal antenna arrays provide powerful performance capabilities whenever an antenna has to fit in geometrically on 3D-shaped surfaces like that found on space- and aircraft. Traditional planar and reflector antenna arrays affect the aerodynamic performance of the aircraft as well as have limited scanning capabilities (usually up to $\pm 60^{\circ}$ ) [1]. Therefore, conformal antenna arrays are considered as an attractive alternative. Conformal array technology is still rarely used in commercial applications. It is limited mostly to military communications and aviation, missile electronic warfare, and radar systems. This is also important for future aircraft designs that use thrust vectoring and, thus, can decouple the flight and aircraft axes. In addition to military applications, the conformal antenna arrays are gaining importance in mobile communications. Future mobile base stations can be concealed for aesthetical reasons. One of the most important applications of the conformal arrays in the future is expected to be the smart skin approach [2]. It envisages the integration of nonplanar active antenna apertures in curved surfaces adapted to the skin of the platform: aircraft, satellite, car, etc. Biomedical applications have been also considered recently for both inbody [3] and on-body [4] devices.

A fundamental problem in the analysis of conformal arrays is that there is an infinite number of the possible surfaces to conform to. Furthermore, if there is no symmetry, each

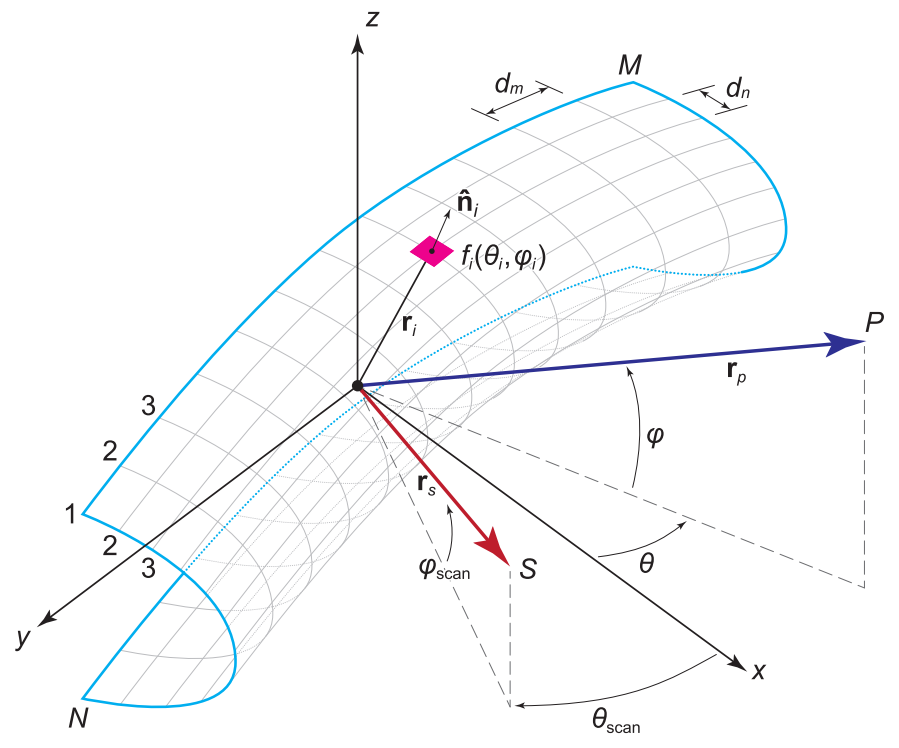

Fig. 1. Problem formulation: an arbitrary shaped surface containing a conformal antenna array defined in a global Cartesian coordinate system as $\left\{\left(\mathbf{r}_{1}, \ldots, \mathbf{r}_{i}\right) \mid \mathbf{r}_{i}\right.$ in $\mathbb{R}^{3}$ and $\left.i=N \times M\right\}$. Individual element orientations are defined using normals $\hat{\mathbf{n}}_{i}$. Vector $\mathbf{r}_{p}$ leads to the observation point in the far field and $\mathbf{r}_{s}$ to the scan direction.

element will see a different environment and this complicates the mathematics. As a consequence, the element factor cannot be factored out from the array factor. This is directly translated to the simulation time needed for the analysis.

Implementation of analysis algorithms is nontrivial as well. It requires interdisciplinary background in antennas and electromagnetics, analytic geometry, advanced linear algebra, and rapid code development. Therefore, only a few codes have been proposed so far. Brégains et al. proposed a methodology for the analysis of arbitrary conformal arrays [5]. The local coordinate systems have to be pre-specified explicitly for each array element. This code was later extended to deal with heterogeneous elements with potentially different polarizations [6]. Kobayashi et al. have recently proposed a simple calculation method of equi-phase fronts of conformal arrays based on the direction-cosine relation [7]. As for the commercial codes, 
to the best of authors' knowledge, only Phased Array System Toolbox ${ }^{\mathrm{TM}}$ implemented in MATLAB [8] provides a complete script-based framework for the analysis of conformal arrays.

In this study, we propose a new tool for rapid analysis of arbitrary-shaped conformal beam-scanning arrays. Compared to [6], only the element position vectors $\mathbf{r}_{i}$ and normals $\hat{\mathbf{n}}_{i}$ are required to set up the array. More than a twofold reduction of execution time and memory complexity was achieved compared to [8]. This enables the analysis of larger and more complex structures. Finally, an example study is provided that examines beam-steering performance of a halfcylinder conformal array $v s$ a reference square planar array.

\section{Problem Formulation}

\section{A. Coordinate Systems}

We define the array geometry in global coordinates (GCS) via the element position vectors $\mathbf{r}_{i}=\left(x_{i}, y_{i}, z_{i}\right)$ and the normals $\hat{\mathbf{n}}_{i}=\left(\theta_{i}, \varphi_{i}\right)$ (Fig. 1). Transitions between spherical and Cartesian forms are as defined in [9, Sec. 3.5.3.1]. For the isotropic elements [i.e. $f_{i}$ is a scalar constant independent of $(\theta, \varphi)$, defining only GCS is enough. However, for an arbitrary conformal array consisting of directive elements, it is useful to define the local coordinates systems (LCS). We accomplish this by, first, placing $\left(\mathbf{r}_{i}\right)$ and orienting $\left(\hat{\mathbf{n}}_{i}\right)$ the array elements in GCS. Then, we use the rotation matrices $\mathbf{X}$, $\mathbf{Y}$, and $\mathbf{Z}$ (as defined in [9, Sec. 3.5.3.2]) around each axis $(x, y, z)$ in Cartesian coordinates. The full $3 \times 3$ transformation matrix $\mathbf{T}$ is then generated for each element by multiplying successively the rotation matrices:

$$
\mathbf{T}=\mathbf{Z Y X}
$$

Assuming no polarization, the rotation is required only around $y$ - and $z$-axes. $\mathbf{X}$ is therefore an identity matrix, or $\mathbf{T}=\mathbf{Z Y}$.

The transformation matrices allow now to determine the set of observation point vectors $\left\{\left(\mathbf{r}_{p_{1}}, \ldots, \mathbf{r}_{p_{i}}\right) \mid \mathbf{r}_{p_{i}}\right.$ in $\left.\mathbb{R}^{3}\right\}$ in LCS for each element by, first, multiplying $\mathbf{T}$ by a columnvector $r_{p}$ and, second, summing the columns of a resulting matrix $\mathbf{R}$. Finally, $\mathbf{r}_{p_{i}}$ could be converted back to spherical coordinates. Now we can determine the contribution of each element at the direction $\mathbf{r}_{p}$.

\section{B. Far-Field Superposition}

For an arbitrary conformal array depicted in Fig. 1, the radiation pattern is obtained by superposition of contributions of each element [10]:

$$
\mathbf{E}(\theta, \varphi)=\sum_{i=1}^{N \times M} \mathbf{f}_{i} w_{i} e^{-j k \mathbf{r}_{i} \cdot \mathbf{r}_{p}},
$$

where $\theta=$ azimuth angle, $\varphi=$ elevation angle, $\mathbf{f}_{i}=$ individual element radiation patterns, $w_{i}=\left|w_{i}\right| e^{-j \beta_{i}}$ are the complex weights, $\beta_{i}=$ phase of the $i^{t h}$ element, $k=2 \pi / \lambda$ is the wavenumber at the frequency $f, \mathbf{r}_{i}=\left(x_{i}, y_{i}, z_{i}\right)$ is the position vector of the $i^{\text {th }}$ element relative to the center of GCS, and $\mathbf{r}_{p}=$ vector to an arbitrary observation point $P(\theta, \varphi)$.
Individual array elements consist mainly of simple antennas: patches, dipoles, monopoles, etc. Their smooth radiation patterns can be accurately reproduced using simple analytical expressions. For the sake of simplicity, we represent here a generic directive antenna using a second-order cosine:

$$
E_{i}=\cos ^{2} \varphi_{i} \cos ^{2} \theta_{i}
$$

\section{Array Scanning}

Array scanning to an angular direction $\left(\theta_{\text {scan }}, \varphi_{\text {scan }}\right)$ can be accomplished by applying the complex weights $w_{i}$. These are chosen so that the path length differences for the generalized conformal array in Fig. 1 are compensated in order to make the radiated phase fronts from all elements arrive in phase at a given point $S$ in far field. Therefore, the complex weights could be easily found as [10, p. 15]:

$$
w_{i}=a_{i} e^{-j k \mathbf{r}_{i} \cdot \mathbf{r}_{s}},
$$

where $a_{i}$ is the real-valued amplitude of the $i^{\text {th }}$ element. Therefore, the phase $\beta_{i}=\tan ^{-1}\left[\Im\left(w_{i}\right) / \Re\left(w_{i}\right)\right]$ and $\mathbf{r}_{s}$ is the steering vector obtained from $\left(\theta_{\text {scan }}, \varphi_{\text {scan }}\right)$ as

$$
\mathbf{r}_{s}=\left[\begin{array}{c}
-\cos \left(\varphi_{\text {scan }}\right) \cos \left(\theta_{\text {scan }}\right) \\
-\cos \left(\varphi_{\text {scan }}\right) \sin \left(\theta_{\text {scan }}\right) \\
-\sin \left(\varphi_{\text {scan }}\right)
\end{array}\right]
$$

Note that (4) is exact for an array of isotropic elements. For anisotropic elements - as (3) — the solution is approximate with its error proportional to the steering angle.

\section{Directive Properties}

Directive gain is a ratio of the array radiation intensity in a given direction over the radiation intensity of an isotropic source. We calculate it as [11]

$$
D=\frac{4 \pi U(\theta, \varphi)}{P_{\mathrm{rad}}}
$$

where $U(\theta, \varphi)=|\mathbf{E}(\theta, \varphi)|^{2}$ is the radiation intensity and $P_{\text {rad }}$ is the total radiated power. The latter could be obtained by integrating $U$ over the entire solid angle of $4 \pi$ [11, Ch. 2.4]:

$$
P_{\mathrm{rad}}=\oiint_{\Omega} U d \Omega=\int_{0}^{2 \pi} \int_{0}^{\pi} U \sin \theta d \theta d \varphi .
$$

We solve (7) numerically as given in [11, Ch. 2.7].

Finally, the directivity $D_{0}$ of the array is defined as the maximum value of the directive gain $D_{0}=\max [D((\theta, \varphi)]$.

\section{EXAMPLE RESULTS}

Using the outlined methodology, we developed a MATLAB code for the analysis of arbitrary-shaped conformal beamscanning arrays. For demonstration and benchmark purposes, we perform a simple analysis of a half-cylindrical-segment conformal array and compare its beam-steering performance with a reference planar array. For instance, such problem can arise in an aircraft-wing antenna design when facing a decision of mounting a planar array behind an aerodynamic radome or conforming the array to a wing. 


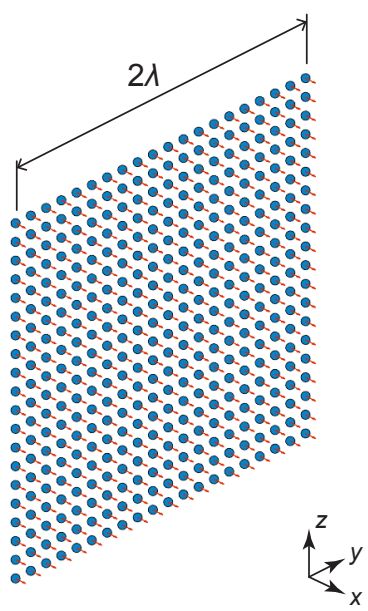

(a)

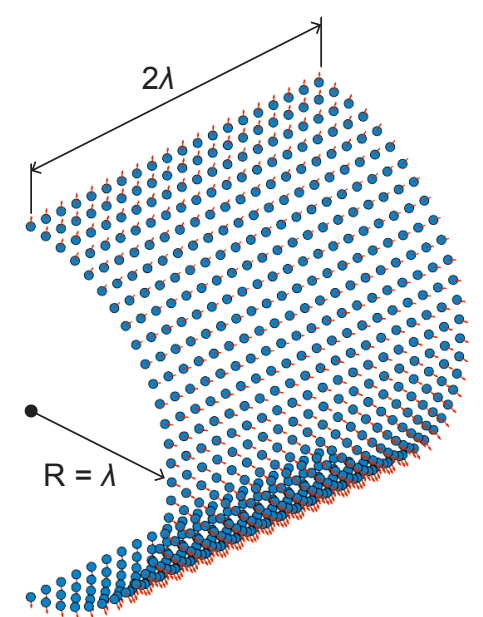

(b)
Fig. 2. Geometry of the example problem: comparison of beam-scanning performance of (a) square planar and (b) half-cylindrical-segment conformal arrays. Both arrays span the same area on $y-z$ plane. The element separation distance is $\lambda / 10$ so that the fundamental bounds on $D_{0}$ are obtained. The individual elements patterns are according to (3). The element normals $\hat{\mathbf{n}}_{i}$ (denoted by red arrows) are orthogonal to the array surfaces.

\section{A. Half-Cylindrical-Segment vs Planar Arrays}

Fig. 2 shows the geometries of the arrays under comparison. The element separation distance is $\lambda / 10$ that allows obtaining the fundamentally achievable $D_{0}$ in a given steering direction $\left(\varphi_{\text {scan }}, \theta_{\text {scan }}\right)$. The square planar array (Fig. 2a) contains 400 elements, and the conformal one (Fig. 2b) has 620 elements. Both arrays span the same $(2 \lambda)^{2}$ area on the $y-z$ plane. The individual element patters $\mathbf{f}_{i}$ are given by (3), and the element normals $\hat{\mathbf{n}}_{i}$ are orthogonal to the array surfaces.

Well-known scanning performance of a planar array is demonstrated in Fig. 3a: the scanning range is limited by a maximum of about $60^{\circ}$ in both azimuth and elevation planes. End-fire radiation in a given direction can only be achieved using the individual elements with appropriate patterns to satisfy the superposition in this direction [12].

Conformal arrays allow to mitigate such limitations. Clearly, the elevation-scan range is improved as Fig. 3b shows: even at $90^{\circ}$ only a few $\mathrm{dBi}$ of scan loss is observed. A less obvious result is that the azimuth scanning range is also significantly improved for non-zero elevation angles. In other words, for the arrays in Fig. 2, the planar one has inaccessible scan range of about $30^{\circ}$ from $y-z$ plane in any direction. However, the half-cylindrical conformal array has a blind zone only $\approx 20^{\circ}$ around the $y$-axis. Note that we analyzed the scanning range only for a $+x$ half-space since we consider here the cosinesquared array elements with no back lobes. The conformal array has also a limited scanning capability in the $-x$ halfspace, i.e. beyond $\varphi_{\text {scan }}>90^{\circ}$. As the scan loss will be high in this angular space, we omitted these results for clarity.

The obtained results were verified using the Phased Array System Toolbox ${ }^{\mathrm{TM}}$ implemented in MATLAB [8] and are in perfect agreement. As for the performance of our code, the

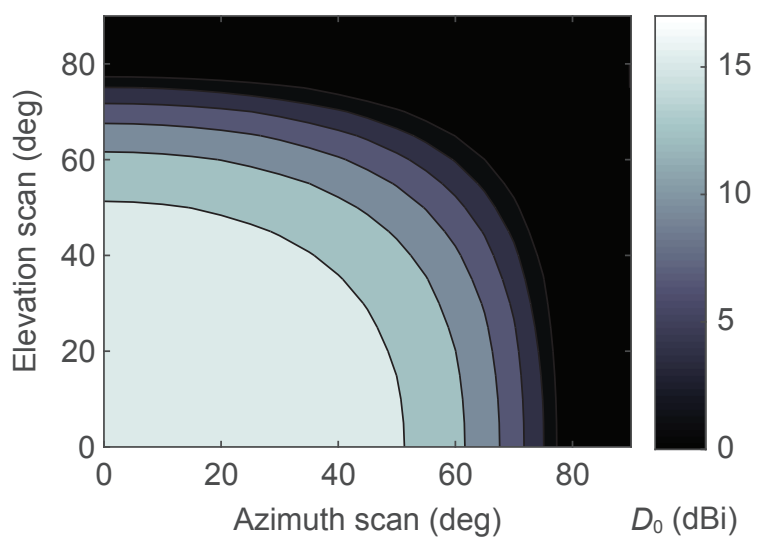

(a)

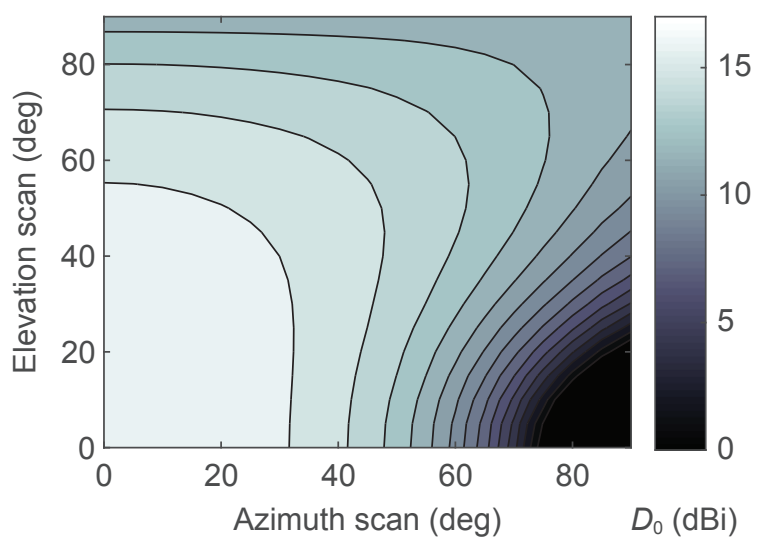

(b)

Fig. 3. Comparison of beam-scanning performance of (a) planar and (b) halfcylindrical-segment conformal arrays. The broadside scanning direction is $(\varphi, \theta)=0^{\circ}$. Only a quarter of the scanning range is shown due to the symmetry properties of the arrays in Fig. 2. Maximum directivity in the scanning direction is shown, and the corresponding elements weights $w_{i}$ are estimated using (4)

analysis of $D_{0}$ for one scanning angle took $9.1 \mathrm{~s}$, whereas [8] requires $21.9 \mathrm{~s}$ to execute (average times over 10 runs; Intel Core i7-6700 CPU, 32 GB DDR4 RAM). Note that $\left(\theta_{\text {scan }}, \varphi_{\text {scan }}\right) \in[0,90]^{\circ}$ has been analyzed to obtain the results in Fig. 3, which represent significant performance improvement.

The memory complexity is also significantly reduced compared to [8]. Low memory usage makes it possible to analyze much larger structures. For instance, the analysis of the $10 \lambda$ half-cylindrical array (15.7k elements) was impossible using [8]: memory overflow occurred even on a computation server with 256 GB RAM. Using our code, the run time for one scanning angle took $48.2 \mathrm{~min}$ in average (over three runs) on the aforementioned 32-GB-RAM desktop computer.

\section{CONCLUSION}

In this study, we proposed a new tool for fast analysis of arbitrary-shaped conformal beam-scanning arrays. Implementation of the proposed methodology allow for rapid and accurate analysis of the radiative properties of conformal 
beam-scanning arrays with arbitrary spatial configurations in $\mathbb{R}^{3}$. Considering nearly identical mathematical approach, the method can also be applied to the analysis of complex 3Dshaped apertures [11, Ch. 12]. Future work includes the implementation of arbitrary element polarizations and existing inhouse optimization approaches (e.g. [13]) to enable radiation pattern synthesis of arbitrary conformal arrays.

\section{REFERENCES}

[1] L. Josefsson and P. Persson, Conformal Array Antenna Theory and Design. Hoboken, N.J.: Wiley-Interscience, 2006.

[2] S. H. Son, S. Y. Eom, and W. Hwang, "Development of a smart-skin phased array system with a honeycomb sandwich microstrip antenna," Smart Mater. Struct., vol. 17, no. 3, p. 035012, Apr. 2008.

[3] D. Nikolayev, M. Zhadobov, P. Karban, and R. Sauleau, "Conforma antennas for miniature in-body devices: The quest to improve radiation performance," URSI Rad. Sci. Bull., vol. 2017, no. 363, pp. 52-64, Dec. 2017.

[4] D. R. Agrawal et al., "Conformal phased surfaces for wireless powering of bioelectronic microdevices," Nat. Biomed. Eng., vol. 1, p. 0043, Mar. 2017.

[5] J. C. Brégains, J. A. García-Naya, A. Dapena, and M. González-López, "A MATLAB tool for visualizing the 3D polar power patterns and excitations of conformal arrays," IEEE Antennas Propag. Mag., vol. 52, no. 4, pp. 127-133, Aug. 2010.

[6] J. C. Brégains, J. A. García-Naya, M. González-López, and L. CastedoRibas, "A MATLAB interface for analyzing conformal arrays composed of polarized heterogeneous elements," IEEE Antennas Propag. Mag., vol. 53 , no. 5 , pp. $136-144$, Oct. 2011.

[7] H. Kobayashi, "Simple calculation method for conformal beam-scanning array pattern," in Proc. 13th Eur. Conf. on Antennas and Propagation (EuCAP 2019), Krakow, Poland, 2019, pp. 1-5.

[8] MathWorks. Phased Array System Toolbox. Accessed: Jul. 26, 2019. [Online]. Available: www.mathworks.com/products/phased-array.html

[9] I. N. Bronshtein, K. A. Semendyayev, G. Musiol, and H. Mühlig, Handbook of Mathematics, 5th ed. Berlin: Springer, 2007.

[10] R. J. Mailloux, Phased Array Antenna Handbook, 3rd ed. Norwood, MA: Artech House, 2018.

[11] C. A. Balanis, Antenna Theory: Analysis and Design, 4th ed. Hoboken, NJ: John Wiley \& Sons, 2016.

[12] J. Liu and Q. Xue, "Microstrip magnetic dipole Yagi array antenna with endfire radiation and vertical polarization," IEEE Trans. Antennas Propag., vol. 61, no. 3, pp. 1140-1147, Mar. 2013.

[13] B. Fuchs, "Shaped beam synthesis of arbitrary arrays via linear programming," IEEE Antenn. Wireless Propag. Lett., vol. 9, pp. 481-484, May 2010. 